\title{
Desempenho Comparativo de Seis Grupos de Cruzamento Holandês/Guzerá. 12. Ganho de Peso de Bezerras e Novilhas ${ }^{1}$
}

\author{
Antonio Aurico Flôres ${ }^{2}$, Fernando Enrique Madalena ${ }^{3}$, Roberto Luiz Teodoro ${ }^{4}$
}

\begin{abstract}
RESUMO - Foram analisados 3.475 pesos de fêmeas de seis grupos genéticos oriundos do acasalamento entre Holandês Vermelho e Branco (HVB) x Guzerá (Guz), com frações esperadas de genes de HVB 1/4, 1/2 ( $\mathrm{F}_{1}$ ), 5/8 (acasalamento inter se), 3/4, 7/8 e $\geq 31 / 32$. Os dados foram analisados separadamente para 374 bezerras (até 120 dias de idade) e 524 novilhas (dos 121 aos 550 dias de idade), por meio do Proc Mixed do pacote SAS, com modelo que incluiu os efeitos fixos de grupo genético (G), trimestre de pesagem, e a idade como co-variável dentro de G, e o efeito aleatório do animal. Aos ganhos de peso foi ajustado modelo aditivo dominante incluindo a diferença aditiva entre as raças $\left(\mathrm{g}^{\mathrm{I}}\right)\left(\right.$ HVB-Guz) e a heterose $\left(\mathrm{h}^{\mathrm{I}}\right)$. Para as bezerras, o $\mathrm{g}^{\mathrm{I}}$ estimado foi significativo $(\mathrm{P}=0,059)$, mas a heterose não ( $\mathrm{P}=0,92)$. O ganho de peso nesta fase foi de $474 \mathrm{~g} / \mathrm{d}$ para as 1/4, de $510 \mathrm{~g} / \mathrm{d}$ para as $1 / 2 \mathrm{e}$ de 575 a $610 \mathrm{~g} / \mathrm{d}$ para os outros quatro grupos, com tendência linear na fração de genes de HVB $\left(\mathrm{g}^{\mathrm{I}}=0,171 \pm 0,065 \mathrm{~g} / \mathrm{d}\right)$, atribuída ao fato observado de as bezerras com maior fração de Bos indicus não se adaptarem bem ao aleitamento em balde. Este menor crescimento foi compensado como novilhas, categoria na qual as $F_{1}$ apresentaram o maior ganho de peso ( $371 \mathrm{~g} / \mathrm{d}$ ), que diminuía para $309 \mathrm{~g} / \mathrm{d}$ nas $1 / 4 \mathrm{e} 256 \mathrm{~g} / \mathrm{d}$ nas $\geq 31 / 32 \mathrm{HVB}$. O modelo aditivodominante predizia adequadamente as taxas de ganho para as novilhas $\mathrm{F}_{1}$ e de retrocruzamentos, mas não para as do cruzamento $5 / 8$ inter se, sugerindo a presença de epistasia. Excluindo-se este último grupo, foram estimados valores de $\mathrm{g}^{\mathrm{I}}=-0,006 \pm 0,023 \mathrm{e}$ $\mathrm{h}^{\mathrm{I}}=0,113 \pm 0,020 \mathrm{~g} / \mathrm{d}$.
\end{abstract}

Palavras-chave: crescimento, cruzamentos, gado de leite, ganho de peso, heterose

\section{Comparative Performance of Six Holstein-Friesian/Guzera Crossbred Groups. Live Weight Gains of Female Calves and Heifers}

\begin{abstract}
The data analyzed were live weights of females (3475) of six genetic groups obtained by crossing red and white Holstein-Friesian (HVB) x Guzerá (Guz), with expected HVB gene fractions 1/4, 1/2 $\left(\mathrm{F}_{1}\right), 5 / 8$ (from inter se mating), 3/4, 7/8 and $\geq 31 / 32$. The data were separately analyzed for 374 calves (up to age $120 \mathrm{~d}$ ) and 524 heifers (121 to 550-d-old), using Proc Mixed of the SAS package, under a model including the fixed effects of genetic group $(\mathrm{G})$, trimester of weighting and age within $\mathrm{G}$ as a co-variable, and animal as a random effect. An additive-dominant model was fitted to the daily gains including the individual breed additive difference ( $\mathrm{g}^{\mathrm{I}}$ ) (HVB-Guz) and heterosis $\left(\mathrm{h}^{\mathrm{I}}\right)$. In calves, the estimated $\mathrm{g}^{\mathrm{I}}$ was significant $(\mathrm{P}=0.059)$ but heterosis was not $(\mathrm{P}=0.92)$. Average daily gain for this category was $474 \mathrm{~g} / \mathrm{d}$ in the $1 / 4,510 \mathrm{~g} / \mathrm{d}$ in the $1 / 2$ and from 575 to $610 \mathrm{~g} / \mathrm{d}$ in the other four groups, with a linear trend on HVB gene fraction $\left(\mathrm{g}^{\mathrm{I}}=0,171 \pm 0,065 \mathrm{~g} / \mathrm{d}\right)$, attributed to the observed fact that the calves with higher Bos indicus fraction did not adapt well to bucket feeding. This lower growth rate was compensated as heifers, category in which the $\mathrm{F}_{1}$ displayed the highest daily weight gain ( $371 \mathrm{~g} / \mathrm{d}$ ), which decreased from $309 \mathrm{~g} / \mathrm{d}$ in the $1 / 4$ to $256 \mathrm{~g} / \mathrm{d}$ in the $\geq 31 / 32 \mathrm{HVB}$. The additive-dominant model adequately fitted the daily gains in $\mathrm{F}_{1}$ and backcross heifers, but not in the $5 / 8$ inter se cross, suggesting the a presence of epistasis. Excluding this last group, estimates were $\mathrm{g}^{\mathrm{I}}=-0,006 \pm 0,023$ and $\mathrm{h}^{\mathrm{I}}=0,113 \pm 0,020 \mathrm{~g} / \mathrm{d}$.
\end{abstract}

Key Words: crossbreeding, dairy cattle, growth, heterosis, weight gain

\section{Introdução}

A taxa de crescimento das fêmeas - característica importante dentro da exploração pecuária de leite está relacionada principalmente com a idade ao primeiro parto. O tipo de cruzamento influencia esta taxa, que, geralmente, apresenta heterose. Em vários trabalhos de cruzamentos entre Holandês e Zebu, os animais $\mathrm{F}_{1}$ apresentaram o maior ganho de peso até os dois anos de idade (Bhat et al.,1978; Saha \& Parekh, 1991; Khanna et al., 1981; Narayanswamy et al., 1984; Roy \& Tripathi, 1991).

A determinação de curvas de crescimento para animais de diferentes categorias econômicas, como bezerras em aleitamento e novilhas de recria, é importante para predizer o peso em diferentes idades.

\footnotetext{
${ }_{1}^{1}$ Parte da Dissertação de Mestrado em Zootecnia do primeiro autor apresentada à UFMG. Trabalho apoiado pelo CNPq e pela CAPES. 2 Fazenda Aimoré, Juti-MS (auricof@yahoo.com.br)

${ }^{3}$ Departamento de Zootecnia, Escola de Veterinária da UFMG, Bolsista do CNPq (fermadal@dedalus.Icc.ufmg.br).

4 EMBRAPA - Gado de Leite (rteodoro@cnpgl.embrapa.br).
} 
Diversos autores têm comunicado relação linear entre o peso e a idade nas fases de cria e recria de fêmeas mestiças leiteiras (Sakhare \& Ingle, 1983; Ganpule et al., 1984; Narayanswamy et al., 1984; Sakhare \& Ingale, 1984; Patel \& Dave, 1987,1988; Saha \& Parekh, 1988).

Objetivou-se, com este estudo, que faz parte do projeto "Estratégias de cruzamentos entre raças leiteiras na Região Sudeste" (Madalena, 1981), ajustar curvas de crescimento para bezerras e novilhas de seis grupos genéticos oriundos do cruzamento entre Holandês Vermelho e Branco x Guzerá, obter estimativas da diferença aditiva entre as raças e da heterose para ganho de peso e testar a validade do modelo aditivo-dominante para esta característica. Modelos de predição do desempenho de diferentes grupos genéticos são úteis para o delineamento de sistemas de cruzamento (Madalena, 2001).

\section{Material e Métodos}

Foram analisados os pesos de 374 bezerras (até 120 dias de idade) e de 524 novilhas (121 a 550 dias de idade), de seis diferentes grupos genéticos Holandês Vermelho e Branco (HVB) x Guzerá (Guz), com frações $1 / 4,1 / 2,5 / 8,3 / 4,7 / 8$ e $\geq 31 / 32$ de HVB. Os grupos serão referenciados por estas frações. Os grupos $1 / 2,3 / 4,7 / 8 \mathrm{e} \geq 31 / 32$ eram $F_{1}$ e retrocruzamentos para HVB, filhas de touros desta raça. Com algumas exceções, os mesmos touros foram utilizados para gerar esses quatro grupos genéticos. As $1 / 4$ eram filhas de touros Guz e as 5/8 eram "bimestiças", filhas de touros e vacas 5/8 HVB/Guz. A amostra dos animais experimentais utilizada no presente estudo foi produzida por meio de inseminação artificial, com sêmen de 24 touros da raça Holandesa e 15 da raça Guzerá, adquiridos de firmas comerciais, e de oito touros 5/8 Holandês x Guzerá, do rebanho da Embrapa. Mais detalhes sobre a origem do rebanho foram apresentados por Lemos et al. (1984).

Os dados utilizados neste estudo foram obtidos no projeto "Estratégias de Cruzamentos entre Raças Leiteiras para a região Sudeste do Brasil”, do ex Centro Nacional de Pesquisa de Gado de Leite (CNPGL), atual Embrapa Gado de Leite. Os animais experimentais foram produzidos e criados na Fazenda Experimental Santa Mônica/Embrapa, Valença - RJ, localizada em região montanhosa, entre 200 e $400 \mathrm{~m}$ de altitude, com clima caracterizado como suave e seco no inverno e quente no verão (Lemos et al., 1984).

As bezerras foram separadas de suas mães logo nos primeiros dias de vida. Foram mantidas em piquetes de capim Bermuda (Cynodon dactylon) e alimentadas, artificialmente, com quatro litros de leite animal/dia, em balde. O concentrado foi oferecido em cochos coletivos, limitado ao consumo médio máximo de $2 \mathrm{~kg}$ por cabeça/dia até os seis meses de idade. Os animais, após 120 dias de idade, foram mantidos em pastagens em que predominava o capim-gordura (Melinis minutiflora). Durante o período da estação seca e outros períodos de escassez,

Tabela 1 - Distribuição do número de animais e observações por grupo genético

Table 1 - Distribuction of animals and records by gentic group

\begin{tabular}{|c|c|c|c|c|c|c|c|}
\hline & \multicolumn{6}{|c|}{$\begin{array}{l}\text { Grupo genético } \\
\text { Genetic group }\end{array}$} & \multirow[t]{2}{*}{ Total } \\
\hline & $1 / 4$ & $1 / 2$ & $5 / 8$ & $3 / 4$ & $7 / 8$ & $\geq 31 / 32$ & \\
\hline \multicolumn{8}{|l|}{ Bezerras } \\
\hline $\begin{array}{l}\text { Female calves } \\
\text { № animais } \\
\text { N. animals }\end{array}$ & 72 & 57 & 56 & 59 & 71 & 59 & 374 \\
\hline $\begin{array}{l}\text { Pesos/animal }{ }^{1} \\
\text { Records/animal }^{1} \\
\text { Novilhas } \\
\text { Heifers }\end{array}$ & 2,0 & 1,8 & 1,9 & 2,0 & 2,0 & 2,0 & 2,0 \\
\hline $\begin{array}{l}\text { № animais } \\
N \text {. animals }\end{array}$ & 96 & 90 & 83 & 86 & 88 & 81 & 524 \\
\hline $\begin{array}{l}\text { Pesos/animal }{ }^{1} \\
\text { Records/animal }^{1}\end{array}$ & 5,3 & 4,8 & 5,2 & 4,9 & 5,8 & 5,3 & 5,2 \\
\hline
\end{tabular}

\footnotetext{
${ }^{1}$ Amplitude, bezerras, 1 a 4 , novilhas 1 a 12 .
}

${ }^{1}$ Range, calves, 1 to 4 , heifers, 1 to 12 .

R. Bras. Zootec., v.33, n.6, p.1695-1702, 2004 (Supl. 1) 
as novilhas receberam capim-elefante (Pennisetum purpureum) picado e, ocasionalmente, 1 a $2 \mathrm{~kg}$ de concentrado comercial, mais mistura mineral ad libitum (Teodoro et al., 1984).

Neste estudo, foram utilizados os dados de pesagens das bezerras e novilhas no período de agosto de 1977 a abril de 1983 . Até setembro de 1978, os animais foram pesados com intervalos mensais e, posteriormente, a intervalos trimestrais, nos meses de janeiro, abril, julho e outubro.

Os dados de bezerras ( 0 a 120 dias de idade) e novilhas (121 a 550 dias de idade) foram analisados em separado. As distribuições dos animais e das observações por grupo genético podem ser observados na Tabela 1.

Os dados foram agrupados em quatro trimestres de pesagem, a partir de janeiro a março. Para considerar heterogeneidade da variância nas análises (ver mais adiante), as observações foram agrupadas em três classes de idade à pesagem: 1) até 50 dias, 2) de 51 a 100 dias e 3) 101 a 120 dias, para as de bezerras, e 1) de 121 a 265 dias, 2) de 266 a 410 dias e 3) de 411 a 550 dias, para as novilhas.

Os dados foram analisados por meio do PROC MIXED, do pacote SAS (1995), apropriado para análise de medidas repetidas (Littell et al., 1996). Com base no modelo $\mathbf{Y}=\mathbf{X B}+\mathbf{Z u}+\mathbf{e}$, em que $\mathbf{Y}$ representa uma matriz de pesos dos animais; $\mathbf{B}$, um vetor desconhecido de parâmetros de efeitos fixos com matriz de incidência $\mathbf{X}$ conhecida; $\mathbf{u}$, um vetor desconhecido de efeitos aleatórios com matriz conhecida de incidência $\mathbf{Z}$; e e, um vetor de erro aleatório desconhecido, sendo u $\sim \mathrm{MVN}(\mathbf{0}, \mathbf{G})$, e MVN $(\mathbf{0}, \mathbf{R})$ e matriz de co-variância de $\mathbf{Y} \mathbf{V}(\mathbf{Y})=\mathbf{Z G Z}+\mathbf{R}$. Foi adotada a estrutura de co-variância espacial SP (pow), que permite modelar a co-variância de medidas repetidas com intervalos irregulares entre elas (Littell et al., 1996), em que $\operatorname{cov}\left(e_{i}, e_{j}\right)=\sigma^{2} \rho^{\text {dij }}$, em que $d_{i j}$ é a distância entre as medidas repetidas. Essa estrutura foi comparada à de simetria composta (CS), em que $\rho=1$.

Após análises preliminares, adotou-se o seguinte modelo estatístico, igual para bezerras e novilhas:

$\mathrm{Y}_{\mathrm{ijkl}}=\mathrm{b}_{0}+\mathrm{G}_{\mathrm{i}}+\operatorname{tri}_{\mathrm{j}}+\mathrm{a}_{\mathrm{ki}}+\mathrm{b}_{\mathrm{i}} \mathrm{x}_{\mathrm{ijkl}}+e_{\mathrm{ijkl}}$

em que $Y_{\mathrm{ijkl}}=1^{\text {-ésimo }}$ peso da $\mathrm{k}^{\text {-ésima }}$ fêmea, no $\mathrm{j}$-ésimo trimestre de pesagem, do $\mathrm{i}^{-}$ésimo grupo genético; $\mathrm{b}_{0}=$ intercepto; $\mathrm{G}_{\mathrm{i}}=$ efeito do i-ésimo grupo genético $(\mathrm{i}=1, \ldots, 6) ;$ tri $_{\mathrm{j}}=$ efeito do $\mathrm{j}$-ésimo trimestre de pesagem $(\mathrm{j}=1, \ldots, 4) ; \mathrm{a}_{\mathrm{ki}}=$ efeito aleatório do $\mathrm{k}^{\text {-ésimo }}$ animal dentro de grupo genético; $b_{i}=$ coeficiente de regressão linear do peso sobre à idade para o $\mathrm{i}^{-}{ }^{- \text {ésimo }}$ grupo genético; $\mathrm{x}_{\mathrm{ijkl}}=$ idade do animal à pesagem, em dias; $e_{\mathrm{ijkl}}=$ erro aleatório residual associado a cada observação.

As regressões $b_{i}$ estimam a taxa de ganho diário para cada grupo genético.

Foram modeladas variâncias diferentes em cada uma das três classes de idade descritas anteriormente para cada categoria. Para testar a significância dos efeitos fixos, utilizou-se a opção DDFM=SATTERTH do PROC MIXED, que calcula os graus de liberdade para testes $\mathrm{F}$ aproximados, considerando-se a distribuição dos efeitos aleatórios dentro dos fixos. As médias dos efeitos fixos classificatórios foram comparadas pelo teste de Scheffé (1959).

A heterogeneidade das regressões $b_{i}$, nos diferentes grupos genéticos, foi testada utilizando-se um modelo [2], igual ao [1], mas com $b_{i}$ substituído por $\mathrm{b}+\mathrm{d}_{\mathrm{i}}$, em que $\mathrm{d}_{\mathrm{i}}$ representa o desvio, no iésimo grupo genético, do coeficiente comum $b$,

$\mathrm{Y}_{\mathrm{ijkl}}=\mathrm{b}_{0}+\mathrm{G}_{\mathrm{i}}+\operatorname{tri}_{\mathrm{j}}+\mathrm{a}_{\mathrm{ki}}+\left(\mathrm{b}+\mathrm{d}_{\mathrm{i}}\right) \mathrm{x}_{\mathrm{ijkl}}+e_{\mathrm{ijkl}}$

Para cada categoria, separadamente, foram obtidas estimativas da diferença aditiva entre as raças e da heterose para o ganho de peso diário. Os coeficientes $b_{i}$ do Modelo [1] foram analisados pelo PROC GLM (SAS, 1995) com um modelo aditivo dominante (Madalena, 2001), da seguinte forma:

$\mathrm{b}_{\mathrm{i}} \mathrm{w}_{\mathrm{i}}=\mathrm{c}+\mathrm{g}^{\mathrm{I}} \mathrm{q}_{\mathrm{i}}+\mathrm{h}^{\mathrm{I}} \mathrm{z}_{\mathrm{i}}+e_{\mathrm{i}}$

em que $\mathrm{w}_{\mathrm{i}}=1 / \operatorname{Var}(\mathrm{bi})$, da análise como o modelo [1]; $\mathrm{c}=$ intercepto; $\mathrm{g} \mathrm{I}=$ diferença aditiva individual entre as raças; $\mathrm{q}_{\mathrm{i}}=$ proporção esperada de genes da raça Holandesa nos indivíduos do i-ésimo grupo genético (assumida $=1$ para o grupo $\geq 31 / 32) \quad(i=1, \ldots, 6)$; $\mathrm{h}^{\mathrm{I}}=$ heterose individual; $\mathrm{z}_{\mathrm{i}}=$ proporção esperada de locos ocupados com um gene de cada raça nos indivíduos do i-ésimo grupo genético $(0,5,1,0,46875$, $0,5,0,25$ e 0 , respectivamente, para os grupos $1 / 4$, $1 / 2,5 / 8,3 / 4,7 / 8$ e $\geq 31 / 32$ ).

Para testar a validade do modelo aditivodominante, os valores preditos com este modelo $\left(b^{*}{ }_{i}=c+g^{I} q_{i}+h^{I} z_{i}\right)$ foram utilizados para corrigir os pesos para idade, $\mathrm{Y}^{\mathrm{c}}=\mathrm{Y}-\mathrm{b}_{\mathrm{i}}{ }_{\mathrm{x}} \mathrm{x}$, sendo, então, esses pesos corrigidos re-analisados sob o modelo [2]. Se o modelo aditivo-dominante se ajustasse bem

R. Bras. Zootec., v.33, n.6, p.1695-1702, 2004 (Supl. 1) 
aos ganhos diários, a variação em $\mathrm{Y}^{\mathrm{c}}$ atribuída à idade e à interação de idade $\mathrm{x}$ grupo genético teria sido retirada, não sendo, portanto, significativa.

\section{Resultados e Discussão}

A heterogeneidade das regressões do peso sobre a idade entre os seis grupos genéticos foi testada pelo modelo [2], e o resultado encontrado foi altamente significativo para ambas as fases de crescimento estudadas $(\mathrm{P}<0,001)$. Assim, os grupos genéticos apresentaram taxas de crescimento (ganho de peso diário) diferentes, justificando a adoção do modelo [1].

A estrutura de co-variância espacial adotada (SP pow) teve melhor ajuste, segundo os testes de Informação de Akaike (AIC) e o Critério Bayesiano de Schwartz (SBC), quando comparada à estrutura simétrica composta (CS), a qual supõe ser constante a correlação entre as medidas repetidas no mesmo animal. Para as estruturas SP e CS, os valores de AIC foram $-2.558,7$ e $-2.627,8$, respectivamente, para as bezerras, e $-11.498,2$ e $-11.757,7$, para as novilhas. Os valores de SBC, na mesma ordem, foram $-2.574,7 \mathrm{e}-2.641,6$, para as bezerras, e $11.518,9 \mathrm{e}-11.775,4$, para as novilhas.

Os testes de $\mathrm{F}$ para os efeitos fixos são apresentados na Tabela 2 e as médias estimadas, na Tabela 3. Apenas o grupo genético, para as bezerras, não foi significativo $(\mathrm{P}>0,05)$.

$\mathrm{Na}$ fase de aleitamento, as bezerras $1 / 4$ e $1 / 2$ apresentaram as menores taxas de ganho de peso diário (Tabela 3). Black $(1982,1984)$ e Ward et al. (1983) relataram que animais de origem Bos indicus apresentaram dificuldades de adaptação à ingestão de leite em balde e, conseqüentemente, menor taxa de crescimento no sistema de criação artificial, uma vez que o ganho de peso vivo é proporcional à quantidade de leite ingerida, segundo o primeiro autor. No presente trabalho, foi notória a dificuldade de adaptação ao aleitamento em balde das bezerras com maior fração de Guzerá, especialmente as 1/4. As bezerras $1 / 2$ apresentaram menor peso ao nascimento, em razão do efeito materno negativo das mães Guzerá (Lemos et al., 1984), mas ultrapassaram as $1 / 4$ na fase de aleitamento (Figura 1). Os outros quatro grupos apresentaram taxas de ganho e pesos médios semelhantes nesta fase (Tabela 3; Figura 1).

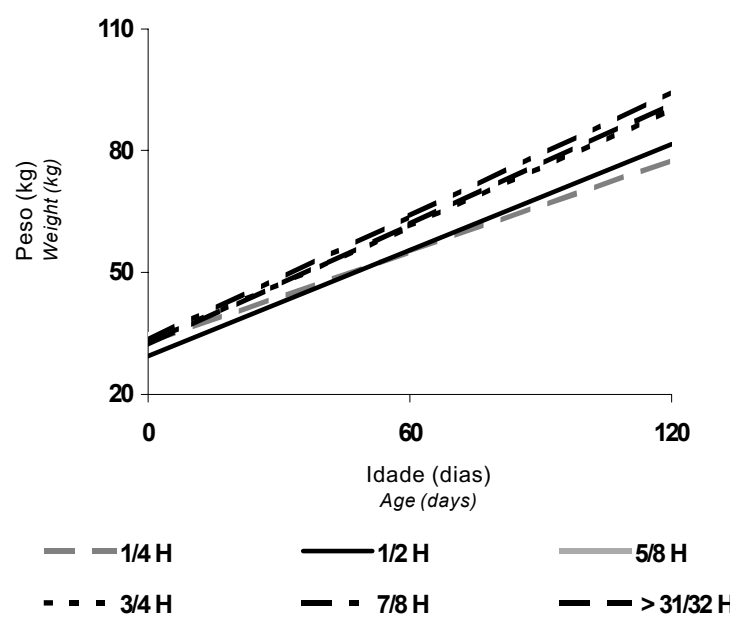

Figura 1 - Crescimento ponderal de bezerras de seis grupos genéticos Holandês/Guzerá.

Figure 1 - Growth of heifer calves of six Hostein-Friesian/ Guzerá crossbred groups.

Tabela 2 - Teste $\mathrm{F}$ dos efeitos fixos nas análises de co-variância sob o modelo [1] Table 2 - F-test for fixed effects in the covariance analyses under model [1]

\begin{tabular}{|c|c|c|c|c|c|c|c|}
\hline & \multicolumn{3}{|c|}{$\begin{array}{c}\text { Bezerras } \\
\text { Female calves }\end{array}$} & \multicolumn{3}{|c|}{$\begin{array}{c}\text { Novilhas } \\
\text { Heifers }\end{array}$} & \multirow[b]{2}{*}{$\operatorname{Pr}>F$} \\
\hline & $\begin{array}{l}\text { GL } \\
D F\end{array}$ & $\mathrm{GL}_{\text {den }}{ }^{1}$ & $\mathrm{~F}$ & $\operatorname{Pr}>F$ & $\mathrm{GL} \mathrm{den}^{1}$ & $\mathrm{~F}$ & \\
\hline $\begin{array}{l}\text { Grupo genético }(\mathrm{G}) \\
\text { Genetic group }(G)\end{array}$ & 5 & 2,3 & 0,24 & 0,916 & 9,44 & 14,13 & 0,000 \\
\hline $\begin{array}{l}\text { Trimestre } \\
\text { Trimester }\end{array}$ & 3 & 6,48 & 7,98 & 0,013 & 672 & 18,75 & 0,000 \\
\hline $\begin{array}{l}\text { Idade/G } \\
\text { Age/G }\end{array}$ & 6 & 49,1 & 745,79 & 0,000 & 1046 & 1768,80 & 0,000 \\
\hline
\end{tabular}

${ }^{1} \mathrm{GL}$ den = graus de liberdade do denominador.

${ }^{1} \mathrm{GL}$ den = denominator degrees of freedom.

R. Bras. Zootec., v.33, n.6, p.1695-1702, 2004 (Supl. 1) 
Tabela 3 - Médias do peso corporal $(\mathrm{kg})$ e coeficientes de regressão $\left(b_{i}\right) \pm$ desvio-padrão (DP), estimados sob o modelo [1]

Table 3 - Means and regression coefficientst \pm standard error (SE), (kg) estimated under model [1]

\begin{tabular}{|c|c|c|}
\hline $\begin{array}{l}\text { Trimestre } \\
\text { Trimester }\end{array}$ & $\begin{array}{c}\text { Bezerras } \\
\text { Female calves }\end{array}$ & $\begin{array}{l}\text { Novilhas } \\
\text { Heifers }\end{array}$ \\
\hline 1 & $63,18 \pm 0,67^{\mathrm{a}}$ & $145,23 \pm 0,94^{\mathrm{c}}$ \\
\hline 2 & $61,53 \pm 0,89^{a, b}$ & $149,73 \pm 0,94^{\mathrm{a}}$ \\
\hline 3 & $63,32 \pm 0,75^{\mathrm{a}}$ & $148,32 \pm 0,96^{\mathrm{b}}$ \\
\hline \multirow[t]{2}{*}{4} & $60,14 \pm 0,59^{b}$ & $145,46 \pm 1,11^{\mathrm{c}}$ \\
\hline & \multicolumn{2}{|c|}{ Grupo genético } \\
\hline $1 / 4$ & $56,20 \pm 0,94^{b}$ & $138,68 \pm 2,01^{\mathrm{c}}$ \\
\hline $1 / 2$ & $58,38 \pm 1,09^{b}$ & $155,44 \pm 2,09^{a}$ \\
\hline $5 / 8$ & $63,71 \pm 1,08^{a}$ & $145,11 \pm 2,16^{\mathrm{b}}$ \\
\hline $3 / 4$ & $63,68 \pm 1,04^{a}$ & $150,95 \pm 2,15^{\mathrm{a}, \mathrm{b}}$ \\
\hline $7 / 8$ & $65,83 \pm 0,96^{\mathrm{a}}$ & $148,23 \pm 2,09^{\mathrm{a}, \mathrm{b}}$ \\
\hline \multirow[t]{2}{*}{$\geq 31 / 32$} & $64,46 \pm 1,04^{\mathrm{a}}$ & $144,70 \pm 2,20^{\mathrm{c}, \mathrm{b}}$ \\
\hline & \multicolumn{2}{|c|}{$\begin{array}{l}\text { Taxa de ganho diário }\left(\mathrm{b}_{\mathrm{i}}\right) \\
\text { Daily gain }\left(b_{i}\right)\end{array}$} \\
\hline $1 / 4$ & $0,474 \pm 0,017$ & $0,309 \pm 0,007$ \\
\hline $1 / 2$ & $0,510 \pm 0,020$ & $0,371 \pm 0,007$ \\
\hline $5 / 8$ & $0,602 \pm 0,020$ & $0,281 \pm 0,007$ \\
\hline $3 / 4$ & $0,575 \pm 0,019$ & $0,294 \pm 0,007$ \\
\hline $7 / 8$ & $0,617 \pm 0,019$ & $0,275 \pm 0,006$ \\
\hline$\geq 31 / 32$ & $0,588 \pm 0,017$ & $0,256 \pm 0,007$ \\
\hline \multicolumn{3}{|c|}{$\begin{array}{l}a, b, c \text { Médias com sobrescritos diferentes indicam diferença } \\
\text { significativa }(P<0,05) \text {. As diferenças entre as regressões } \\
\text { não foram testadas individualmente. }\end{array}$} \\
\hline \multicolumn{3}{|c|}{$\begin{array}{l}\text { Means with different superscript differ significantly }(P<.05) \text {. The } \\
\text { significance of differences between regressions was not } \\
\text { individually tested. }\end{array}$} \\
\hline
\end{tabular}

$\mathrm{Na}$ fase de crescimento a pasto, após o desaleitamento, as novilhas $\mathrm{F}_{1}$ apresentaram as maiores taxas de ganho, compensando o menor crescimento como bezerras (Tabela 3; Figura 2). Diversos autores relataram maiores ganhos de peso de novilhas $\mathrm{F}_{1}$ em comparações de diversos cruzamento entre Holandês x Zebu (Bhat \& Singh, 1978; Narayanswamy et al., 1984; Sakhare \& Ingale, 1984; Saha \& Parekh, 1988, Jadhav et al., 1991; Roy \& Tripathi, 1991). As novilhas $\mathrm{F}_{1}$ deste projeto continuaram apresentando maior ganho de peso em fase posterior, após distribuição em fazendas colaboradoras (Madureira et al., 2002).

\section{Modelos genéticos}

Ao se ajustar o modelo aditivo-dominante [3] aos ganhos diários nas bezerras ( $b_{i}$ do modelo [1]), apenas a diferença aditiva entre as raças foi significativa, mas não o foi a heterose, $(P=0,92)$, sendo, portanto, retirada do modelo para esta categoria. Os valores de c e $\mathrm{g}^{\mathrm{I}}$ são apresentados na Tabela 4.

R. Bras. Zootec., v.33, n.6, p.1695-1702, 2004 (Supl. 1)
Tabela 4 - Intercepto (c), diferença aditiva entre as raças (Holandês menos Guzerá, $g^{\prime}$ ) e heterose individual $\left(h^{l}\right)$ para ganho de peso \pm desviopadrão

Table 4 - Intercept (c), aditive breed difference (Holstein minus Guzera, $g^{\prime}$ ) and individual heterosis $\left(h^{l}\right)$ for weight gain \pm standard error

\begin{tabular}{lcc}
\hline & Bezerras & Novilhas $^{1}$ \\
& Female calves & Heifers $^{1}$ \\
\hline $\mathrm{C}$ & $0,454 \pm 0,046^{* *}$ & $0,255 \pm 0,023 * *$ \\
$\mathrm{~g}$ & $0,171 \pm 0,065^{*}$ & $-0,006 \pm 0,023 \mathrm{~ns}$ \\
$\mathrm{~h}^{\mathrm{I}}$ & - & $0,113 \pm 0,020 * *$ \\
\hline
\end{tabular}

ns $P>0,05 ;{ }^{*} \mathrm{P}=0,059 ;{ }^{* *} \mathrm{P}<0,05$.

1 Valores estimados para as progênies de pais puros (as bimestiças $5 / 8$ foram excluídas da análise, ver texto).

ns $P>.05 ;$ * $P=.059$; ** $(P<.05)$.

${ }^{1}$ Pure parents offspring estimated values (the $5 / 8$ was bimestizo excluded of the analysis, see the text).

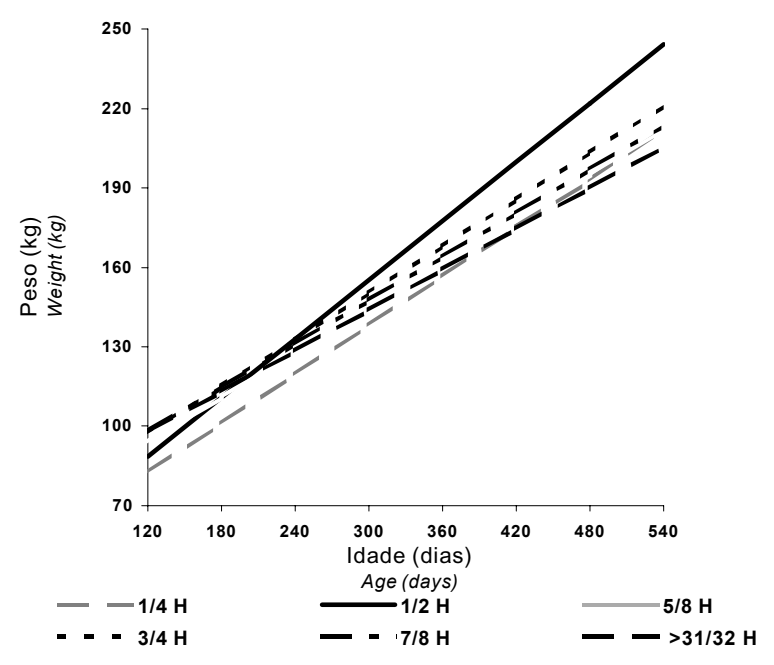

Figura 2 - Crescimento ponderal de novilhas de seis grupos genéticos Holandês/Guzerá.

Figure 2 - Growth of heifers of six Hostein-Friesian/Guzerá crossbred groups.

$\mathrm{Na}$ análise dos pesos corrigidos para idade $\left(\mathrm{Y}^{\mathrm{c}}=\mathrm{Y}-\mathrm{b}^{*}{ }_{\mathrm{i}} \mathrm{x}, \mathrm{b}^{*}{ }_{\mathrm{i}}=\mathrm{c}+\mathrm{g}^{\mathrm{I}} \mathrm{q}_{\mathrm{i}}\right)$ com o modelo [2], a regressão média sobre a idade não foi significativa $(\mathrm{P}=0,44)$, mas a heterogeneidade das regressões nos seis grupos genéticos o foi $(\mathrm{P}=0,03)$, indicando que o modelo aditivo-dominante [3] prediz adequadamente a taxa de ganho média para os seis grupos genéticos das bezerras, porém não eliminava totalmente a variação das taxas de ganho entre os grupos. As regressões do peso corrigido sobre a idade não foram significativamente diferentes de zero para os grupos 
$1 / 4,1 / 2$ e $3 / 4(\mathrm{P}>0,23)$, mas para os grupos $5 / 8,7 / 8$ e $\geq 31 / 32$ tiveram valores de $b_{i}=0,042 ; 0,013 \mathrm{e}-0,037$, respectivamente $(\mathrm{P}<0,04)$.

Os valores esperados de ganho de peso para os diferentes grupos genéticos, calculados pelo modelo aditivo-dominante, e as médias obtidas pelo modelo classificatório [1] podem ser visualizados na Figura 3, em que se observa a relação linear do ganho com a fração esperada de genes da raça Holandesa.

Para as novilhas, a heterose no ganho de peso foi significativa $(\mathrm{P}=0,03)$ e a diferença aditiva entre as raças (HVB menos Guz) não ( $\mathrm{P}=0,81)$ (Tabela 4).

A regressão dos pesos corrigidos para idade $\left(\mathrm{Y}^{\mathrm{c}}=\mathrm{Y}-\mathrm{b}_{\mathrm{i}}{ }_{\mathrm{i}} \mathrm{x}\right)$ sobre a idade foi significativa $(\mathrm{P}=0,0009)$ no modelo [2], assim como a heterogeneidade das regressões nos seis grupos genéticos $(P=0,01)$, indicando que os coeficientes $\left(b^{*}{ }_{i}=c+g^{I} q_{i}+h^{I} z_{i}\right)$, preditos com o modelo aditivodominante [3], não removiam tais efeitos, ou seja, o modelo aditivo-dominante não era apropriado para predizer as regressões $b_{i}$ do modelo [1]. Em razão de a epistasia ser uma causa possível de falta de ajuste do modelo aditivo-dominante e, entre os grupos genéticos deste experimento, somente o 5/8 ser "bimestiço", isto é, de acasalamento inter se de pais e mães mestiços, com maior recombinação genética

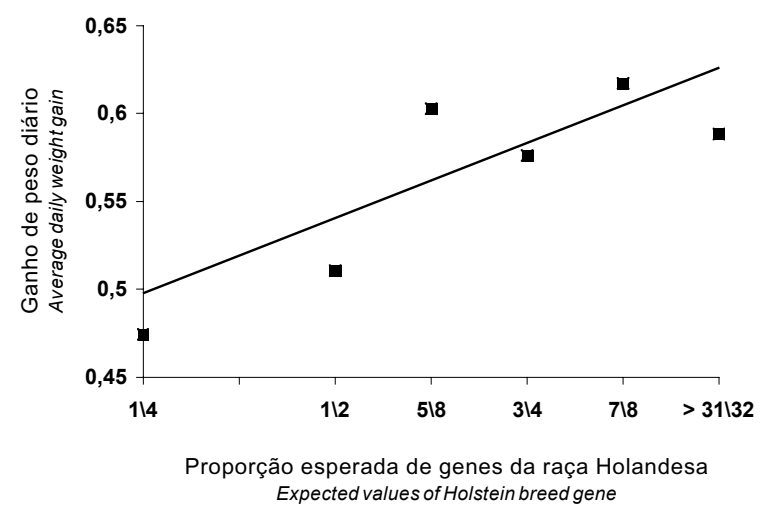

Figura 3 - Ganho de peso diário para as bezerras de até 120 dias de idade. A linha contínua representa os valores esperados sob o modelo aditivodominante e o símbolo $\mathbf{\square}$, as médias para os grupos genéticos no modelo de classificação.

Figure 3 - Weight gain of female calves up to age $120 \mathrm{~d}$. The line represents the expected values under the additive-dominance, and the symbol represents the genetic groups means under the classification model.

R. Bras. Zootec., v.33, n.6, p.1695-1702, 2004 (Supl. 1) que os outros cinco grupos, o modelo [3] foi substituído por um modelo similar [4], em que $\mathrm{b}^{* *}{ }_{\mathrm{i}}=\mathrm{c}_{1}+\mathrm{g}_{1}{ }_{1}$ $\mathrm{q}_{\mathrm{i}}+\mathrm{h}^{\mathrm{I}}{ }_{1} \mathrm{z}_{\mathrm{i}}$ para as filhas de pais puros ou $\mathrm{b}^{* *}{ }_{\mathrm{i}}=\mathrm{b}_{5 / 8}$ para as bimestiças, ou seja, o modelo aditivo-dominante foi ajustado às regressões $b_{i}$ do modelo [1], excluindo-se o grupo 5/8 da análise. Assim, para os retrocruzamentos e as $\mathrm{F}_{1}$, os $\mathrm{b}^{* *}$ i eram os $\mathrm{b}$ preditos com o modelo aditivo-dominante e, para as 5/8, o $b_{i}$ estimado com o modelo [1]. Ao se analisarem os pesos corrigidos desta forma $\left(\mathrm{Y}^{\mathrm{c}}=\mathrm{Y}-\mathrm{b}^{* *}{ }_{\mathrm{i}} \mathrm{x}\right)$ com o modelo [2], os efeitos de idade e a heterogeneidade das regressões entre os grupos genéticos não mais foram significativos $(\mathrm{P}=0,98$ e $\mathrm{P}=0,42)$, indicando que os $b_{i}^{* *}$ removiam as variações nos pesos devido à idade, isto é, o modelo aditivo-dominante se ajustava bem às taxas de ganho nos retrocruzamentos e nas $\mathrm{F}_{1}$, mas não às do cruzamento 5/8 inter se. Fenômeno semelhante foi observado por Madalena et al. (1990), para a produção de leite e caracteres relacionados em animais deste mesmo projeto, o que foi interpretado pelos autores como evidência de epistasia, uma vez que somente nos gametas dos pais do grupo $5 / 8$ inter se haveria recombinação de genes não alélicos, de origem racial diferente. Tal interpretação se aplica também aos presentes resultados.

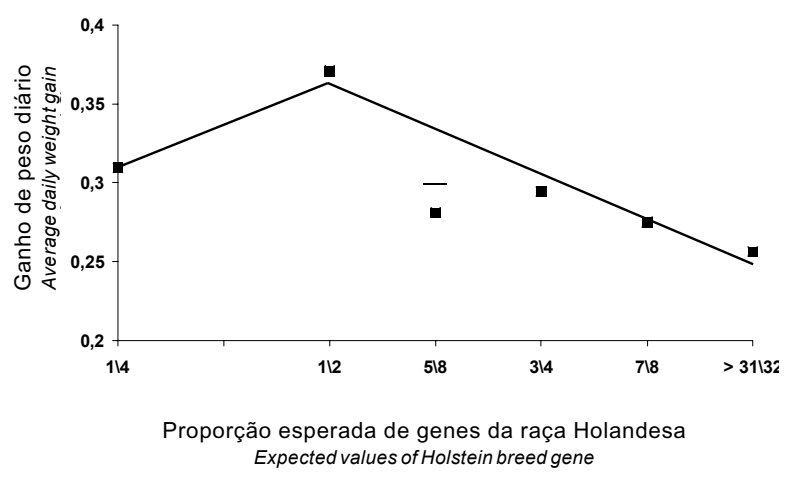

Figura 4 - Ganho de peso diário para as novilhas de 121 a 550 dias de idade. A linha contínua representa os valores esperados sob o modelo aditivo-dominante e o símbolo $\mathbf{\square}$, as médias para os grupos genéticos no modelo de classificação.

Figure 4 - Weight gain of heifers 121 to 550-d-old. The line represents the expected values under the additivedominance, and the symbol represents the genetic groups means under the classification model. 
A estimativa da heterose, que teve importante contribuição sobre o ganho de peso das novilhas, é apresentada na Tabela 4. Heterose importante foi encontrada por Teodoro et al. (1984), para idade e peso à puberdade, e por Madureira et al. (2002), para o peso das novilhas de mais de dois anos de idade, com animais deste mesmo Projeto. Entretanto, o modelo aditivo-dominante explicou adequadamente a variação entre os grupos genéticos, não havendo evidência de epistasia nesses caracteres.

Os valores esperados para ganho de peso das novilhas dos diferentes grupos genéticos, calculados a partir do modelo aditivo-dominante, e as médias de ganho de peso de cada grupo, estimadas pelo modelo classificatório [1], podem ser melhor visualizados na Figura 4, em que se observa o efeito da heterose pelo melhor desempenho apresentado pelas novilhas $1 / 2 \mathrm{e}$ a diminuição no ganho de peso dos animais com o aumento ou a redução da fração de genes de Holandês.

\section{Conclusões}

Tendência linear foi adequada para descrever o crescimento de bezerras e novilhas.

A heterose individual foi muito importante para o crescimento ponderal de novilhas, mas a diferença entre Holandês e Guzerá não diferiu de zero, sendo o $\mathrm{F}_{1}$ o grupo com maior taxa de ganho de peso. Na fase de aleitamento, ao contrário, a heterose não diferiu de zero e o ganho diário foi proporcional à fração de genes de Holandês, refletindo menor adaptação ao aleitamento em balde das bezerras com maior fração de genes de Bos indicus, o que pode ser compensado com um maior ganho na fase pós-aleitamento.

$\mathrm{O}$ modelo aditivo-dominante predizia adequadamente as taxas de ganho para as novilhas $\mathrm{F}_{1}$ e de retrocruzamentos, mas não para as do cruzamento $5 / 8$ inter se, sugerindo a presença de epistasia.

\section{Literatura Citada}

BHAT, P.N.; SINGH, V.P. Effect of genetic and non-genetic factors on body weight in crosses of Hariana with HolsteinFriesian, Brown Swiss and Jersey. Indian Journal of Animal Science, v.48, n.11, p.797-804, 1978.

BHAT, P.N.; TANEJA, V.K; GARG, R.C. Body weight changes in Holstein $\mathrm{x}$ Sahiwal cross-breds in relation to level of Holstein inheritance. Indian Journal of Animal Science, v.48, n.7, p.485-491, 1978.
BLACK, D.H. Calf rearing in the tropics: behavioural responseby Bos indicus calves to an artificial rearing system. Tropical Animal Health and Production, v.16, p.47-48, 1984.

BLACK, D.H. Calf rearing in the tropics: growth rates and utilisation of milk substitute diets. Tropical Animal Health and Production, v.14, p.172-176, 1982.

GANPULE, S.P.; CHAUDHARY, R.P.; TOMAR, S.P.S. Sire $\mathrm{x}$ location interactions in Friesian $\mathrm{x}$ Sahiwal crossbreds under Indian tropical conditions. Indian Journal Dairy Science, v.37, n.1, p.1-4, 1984.

JADHAV, K.L.; TRIPATHI, V.N.; KALE, M.M. Instantaneous per cent growth rate in Holstein Friesian x Sahiwal. Indian Journal Dairy Science, v.44, n.3, p.249-252, 1991.

KHANNA, A.S.; JAISWAL, U.C.; CHOPRA, S.C. et al. Genetic group differences for body weights at various ages in crossbred cattle. Indian Journal Dairy Science, v.34, n.3, p.345-347, 1981.

LEMOS, A.M.; TEODORO, R.L.; BARBOSA, R.T. et al. Comparative performance of six Holstein-Friesian x Guzerá grades in Brazil. 1. Gestation length and birth weight. Animal Production, v.38, p.157-164, 1984.

LITTELL, R.C.; MILLIKEN, G.A.; STROUP, W.W. et al. SAS system for mixed models. Cary: 1996.

MADALENA, F.E. Crossbreeding strategies for dairy cattle in Brazil. World Animal Review, v.38, p.23-30, 1981.

MADALENA, F.E. Consideraciones sobre modelos para la predicción del desempeño de cruzamientos en bovinos. Archivo Latinoamericano Producción Animal, v.9, n.2, p.108-117, 2001

MADALENA, F.E.; LEMOS, A.M.; TEODORO, R.L. et al. Dairy production and reproduction in Holstein-Friesian and Guzerá crosses. Journal of Dairy Science, v.73, p.1872-1886, 1990.

MADUREIRA, A.P.; MADALENA, F.E.; TEODORO, R.L. Desempenho comparativo de seis grupos de cruzamento Holandês/Guzerá. 11. Peso e altura de vacas e novilhas. Revista Brasileira de Zootecnia, v.31, n.2, p.658-667, 2002.

NARAYANSWAMY, M.; BALAINE, D.S.; SHRI RAM. Studies on growth efficiency in Friesian x Sahiwal crosses. Indian Journal Dairy Science, v.37, n.1, p.44-49, 1984.

PATEL, A.M.; DAVE, A.D. Effect of Inter se mating on growth rate and feed conversion efficiency of Holstein Friesian $\mathrm{x}$ Kankrej crossbred calves. Indian Journal Dairy Research, v.7, n.3, p.131-134, 1988.

PATEL, J.M.; DAVE, A.D. Body weight changes and growth pattern in Jersey $x$ Kankrej and Holstein x Kankrej F1 crossbreds. Indian Journal Animal Research, v.21, n.2, p.97-101, 1987.

ROY, P.K.; TRIPATHI, V.N. Studies on body weights up to two years of age in Friesian x Sahiwal/Tharparkar crossbreds. Indian Veterinary Journal, v.68, n.2, p.174-176,1991.

SAHA, D.N.; PAREKH, H.K.B. Factors affecting body weights in half and three fourth crossbred cattle. Indian Journal of Dairy Science, v.41, n.4, p.394-397, 1988.

SAHA, D.N.; PAREKH, H.K.B. Year effect on body weights in crossbreed cattle. Indian Journal of Dairy Science, v.44, n. 7, p.456-458, 1991.

SAKHARE, P.G.; INGALE, U.M. Factors affecting growth rate in Friesian x Sahiwal crossbreds. Indian Veterinary Journal, v.61, n.5, p.414-418, 1984. 
SAKHARE, P.G.; INGLE, U.M. Genetic and non-genetic factors affecting birth weight in Holstein-Friesian $x$ Sahiwal crossbred calves. Indian Journal of Dairy Science, v.36, n.2, p.184-186, 1983.

STATISTICAL ANALYSIS SYSTEM - SAS. User's guide. Cary: 1995.

SCHEFFÉ, H. The analysis of variance. New York: Wiley, 1959. 424p.

TEODORO, R.L.; LEMOS, A.M.; BARBOSA, R.T. et al. Comparative performance of six Holstein-Friesian x Guzerá grades in Brazil - 2. Traits related to the onset of the sexual function. Animal Production, v.38, p.165-170, 1984.
WARD, J.D.B.; BREMNER, K.J.; KILGOUR, R. Behaviour factors influencing the rearing of sahiwal-cross calves. Proceedings of the New Zealand Society Animal Production, v.43, p.171-173, 1983.

Recebido em: 28/07/03

Aceito em: 26/04/04 